\title{
Effects of Dorsal Column Spinal Cord Stimulation on Neuroinflammation: Revisiting Molecular Mechanisms and Clinical Outcomes on Chronic Lumbar/Leg Pain and Failed Back Surgery Syndrome
}

\author{
Marco Echeverria- \\ Villalobos' \\ Justin Mitchell ${ }^{2}$ \\ Juan Fiorda-Diaz' \\ Tristan Weaver'
}

'Department of Anesthesiology, The Ohio State University Wexner Medical Center, Columbus, OH, USA; ${ }^{2}$ College of Medicine, The Ohio State University, Columbus, $\mathrm{OH}$, USA
Correspondence: Tristan Weaver Department of Anesthesiology. The Ohio State University Wexner Medical Center, N4II Doan Hall, 4I0 W I0th Ave,

Columbus, $\mathrm{OH}, 43210$, USA

$\mathrm{Tel}+$ I 614 366-8397

Fax + I 614 366-1943

Email Tristan.Weaver@osumc.edu
Objective: In this narrative review, we reviewed and discussed current literature describing the molecular mechanisms leading to neuroinflammation and its role in the onset and progression of chronic neuropathic lumbar and leg pain in patients with persistent spinal pain syndrome. In addition, we reviewed the proposed mechanisms and impact of spinal cord stimulation (SCS) on neuroinflammation.

Methods: A broad search of current literature in PubMed, Embase, Scopus, Cochrane library, Medline/Ovid, and Web of Science was performed using the following terms and their combinations: "biomarkers", "chronic back and leg pain", “cytokines", "neuroinflammation", "spinal cord stimulation (scs)," and "spinal cord modulation". We selected: 1) articles published in the English language between January 2000 and July 2020 2) preclinical and clinical data 3) case reports 4) meta-analysis and systematic reviews and 5) conference abstracts. Manuscripts not disclosing methodology or without full-text availability were excluded.

Discussion: SCS techniques have gradually evolved since inception to include novel methods such as burst-SCS, high frequency SCS, and differential targeted multiplexed SCS. The incidence of chronic pain after spine surgery is highly variable, with at least one third of patients developing persistent spinal pain syndrome. Novel SCS techniques have been associated with improved clinical and functional outcomes thus increasing patient quality of life.

Conclusion: Currently, health care providers rely on different options and methods for SCS when treating patients with refractory chronic lumbar pain and persistent spinal pain syndrome. Nevertheless, compelling clinical trials remain necessary to elucidate the long-term benefits and mechanisms of neuromodulation of all different types of SCS.

Keywords: neuroinflammation, biomarkers, chronic back and leg pain, cytokines, spinal cord stimulation

\section{Introduction}

Chronic pain is a well-known clinical entity affecting a substantial proportion of the general population. ${ }^{1,2}$ Undoubtedly, chronic pain results in a significant physical, psychological, emotional and economic burden for patients. Chronic low back pain (LBP) comprises a heterogeneous group of disorders including, but not limited to, persistent spinal pain syndrome and radicular pain. ${ }^{3}$

Neuroinflammation, including alteration of glial cells function following nerve injury or gliosis, has emerged as a promising mechanism triggering the transition to chronic back pain. Glial cells such as microglia, oligodendrocytes, astrocytes, and 
ependymal cells have shown to alter activity and gene expression when exposed to physiological stressors, including nerve damage. ${ }^{4,5}$ Preclinical evidence has identified neuroimmune and inflammatory neuromodulatory responses in the nervous system (eg pathological activation of microglia and astrocytes in the spinal cord) as two of the major contributors to pain pathogenesis and persistence. ${ }^{6}$ Both mechanisms have been widely studied in animals laying the groundwork for specific pain therapies in humans. However, the impact of these neuroimmune and neuroinflammatory responses on the onset of acute pain and its transition to chronic entities in humans is yet to be determined. ${ }^{7}$

Initially, the development of novel therapeutic approaches for patients experiencing nociceptive and/or neuropathic chronic pain was driven by the necessity to avoid long-term side effects resulting from the chronic use of nonsteroidal anti-inflammatory drugs (NSAIDs) and opioid therapies. Melzack and Wall originally proposed the gate control theory of pain which led to the current description of the SCS mechanisms of action. ${ }^{8}$ Conventional SCS devices were able to elicit paresthesia through electrical stimulation of the dorsal columns and, since their inception, technical complexity has grown exponentially. ${ }^{9}$ Therefore, early stimulation devices have been continuously updated with adaptive programming and a wide array of new lead placements, frequencies and stimulation patterns. ${ }^{10}$

Cytokines are involved in several nervous system biological processes including neurogenesis, neuron outgrowth, neural survival, and synaptic pruning, transmission and plasticity. ${ }^{11,12}$ In a cross-sectional clinical study, Bäckryd et al reported a significant increase of chemokines levels in cerebrospinal fluid (CSF) of patients with neuropathic pain when compared to healthy controls. ${ }^{13}$ Moreover, significant reduction in CSF concentrations of pro-inflammatory molecules including interleukin (IL) 1, IL-6, tumor necrosis factor alpha (TNF- $\alpha$ ) and vascular endothelial growth factor (VEGF) have been described after spinal cord stimulation (SCS). ${ }^{14}$ Preliminary work by Vallejo et al on differential target multiplexed SCS (DTM-SCS) and an user-adaptive and effective SCS model has shown promising results. ${ }^{15}$ Additionally, Vallejo's group published an optimized algorithm of stimulation that is able to shift glial cell gene expression to a naïve phenotype after sciatic nerve ligation model in rodents. ${ }^{16}$
Recently, the successful implementation of promising therapies that do not rely on paresthesia such as Burst SCS (B-SCS), high frequency SCS (HF-SCS) and differential target multiplexed SCS, has challenged the fundamental gate control theory. ${ }^{17,18}$ Improved sleep, decreased incidence of depression, and sustained reduction in LBP are some of the outcomes associated with these therapies in patients with persistent spinal pain syndrome are. ${ }^{19,20}$

A robust body of preclinical evidence describes the important role of glial cells in the initiation and development of chronic pain. Taken together, it is not unreasonable to consider SCS as a modulatory mechanism of the neuroimmune interface which may result in chronic pain reduction. Although SCS clinical and transitional research continue growing, published reports remain limited.

\section{Methods}

A broad search of current literature in PubMed, Embase, Scopus, Cochrane Library, Medline/Ovid and Web of Science was performed using the following terms and their combinations: "biomarkers", "chronic back and leg pain", “cytokines", "neuroinflammation", "spinal cord stimulation (SCS)" and "spinal cord modulation". We included in our selection: 1) articles published in English language between January 2000 and July 2020 2) preclinical and clinical data 3 ) case reports 4) meta-analysis and systematic reviews and 5) conference abstracts. Preclinical data was mainly discussed when clinical findings on a specific matter were limited. Moreover, manuscripts disclosing no methodology or with no full-text availability were excluded from our narrative review.

\section{Results and Discussion Lumbar/Leg Pain, Failed Back Surgery Syndrome and Neuroinflammation}

The International Association for the Study of Pain (IASP) defines Failed Back Surgery Syndrome (FBSS) as "a spinal pain of unknown origin either persisting despite surgical intervention for spinal pain originally in the same topographical region". ${ }^{21}$ Epidural fibrosis, foraminal stenosis, global or lateral canal stenosis, recurrent disc herniation or degeneration, retained disc fragment, discitis, adhesive arachnoiditis, facet joint pain, spinal instability and sacroiliac joint pain have all been associated with the onset of FBSS. ${ }^{22}$ Reported incidence of chronic pain after spine surgery is highly variable (from $5 \%$ to $75 \%$ ), with 
$20 \%$ to $40 \%$ of these patients fulfilling the clinical criteria for FBSS diagnosis. ${ }^{23}$

Degenerative disc disease (DDD) and lumbar disc herniation are the most common causes of LBP, with both commonly associated with radicular leg pain. ${ }^{24,25}$ High levels of proinflammatory cytokines (eg IL-1 $\beta$ and TNF$\alpha$ ) have been reported in tissue of human degenerative and herniated discs suggesting a regulatory effect in the expression of genes encoding extracellular matrixdegrading enzymes. ${ }^{26}$ Increased activity of matrix metalloproteinase (MMP) leads to rapid degradation of extracellular matrix and proteoglycan loss in the nucleus pulposus (NP), decreasing the weight-bearing capacity and height of the disc. As disc degradation progresses, the annular ring fissures and the NP extrudes, generating pain by compression of the nerve roots. ${ }^{27,28}$ Discogenic pain can also occur in early stages of disc degeneration in the absence of nerve compression and may be exclusively due to the presence of pro-inflammatory cytokines synthesized by intervertebral cells, monocytes and/or macrophages located in the degenerated disc. ${ }^{29,30}$ Lee et al compared the expression of cytokines and growth factors in disc specimens obtained from patients with herniated nucleus pulposus (HNP) and DDD. Authors found a higher expression of IL- 8 , TNF- $\alpha$, transforming growth factor- $\beta$ (TGF- $\beta$ ), VEGF and nerve growth factor (NGF) in disc tissue specimens from patients with DDD when compared to patients with HNP. However, expression of IL-1 $\beta$, IL-6, and IL-12 was comparable among groups. ${ }^{31}$ These results were not consistent with previous findings from cultured NP biopsies describing a higher level of IL6 and IL-8 in painful DDD patients in comparison with $\mathrm{HNP} /$ sciatica patients. $^{32}$

Degenerative disc disease associated pain differs from facet joint pain. The disc lacks synovial structure, an important anatomic component affected by the inflammatory response during osteoarthritis. However, the inflammatory synovial reaction to cartilage damage in the degenerated facet joints leads to increased capsular vascularization with concomitant nociceptors activation and sensitization by cytokines (ie IL-1, IL-6) and inflammatory cells. ${ }^{33,34}$ Local inflammation of the facet joints could spread directly to the dorsal root ganglia (DRG) due to close proximity, affecting neural components (eg neurons and axons) and resulting in radicular pain. ${ }^{35}$ Cytokine, growth factor and prostaglandin release may activate transducer molecules amplifying the current that opens the voltage-gated sodium channels and triggering an action potential that upsurges excitability and signaling in nociceptive neurons. $^{36}$

\section{Chronic Pain and Glial Cells}

Microglia, astrocytes, oligodendrocytes, and ependymal cells are part of the CNS glial cells. Likewise, satellite glial cells (SGC) and Schwann cells are found in the PNS. $^{4,5}$ An important role of the glia is to provide a functional microenvironment that modulates signal transduction, neuroplasticity and synaptic pruning. Direct mechanical trauma (eg, spinal surgery) and peripheral nerve injury results in microglial activation and subsequent increased expression of toll-like receptors (TLR) in the CNS through a mechanism involving pro-inflammatory cytokines such as the high-mobility group box 1 (HMGB1). ${ }^{7}$ Additionally, the increase of TLR4 expression in microglia and macrophages has been associated with greater neuroinflammation and neuronal dysfunction in animal models. ${ }^{37}$ Moreover, impaired cellular signaling, receptor transduction and release of inflammatory mediators may result from morphological glia changes following nerve injury. ${ }^{38}$ Biosynthesis and release dysregulation of inflammatory cytokines such as IL- $1 \beta$, IL- 6 and TNF- $\alpha$, either in the central or peripheral nervous system, have a significant impact on synaptic transmission, neuronal networking excitability and ultimately, in neuropathic pain. $^{39}$

A substantial amount of evidence emphasizes some of the relevant neuromodulatory properties of cytokines in addition to their established actions during infection or trauma. Cytokines are involved in several neural mechanisms such as neurogenesis, neurite outgrowth, neural survival, and synaptic pruning, transmission and plasticity. ${ }^{40}$ Pre-clinical findings have shown that cytokines released by glial cells, neurons, and microvascular endothelial cells, directly modulate neuronal activity through their interaction with cerebral and spinal cord receptors. This process results in activation of ion and voltage-operated channels, and presynaptic neurotransmitter release such as glutamate and gamma aminobutyric acid (GABA). ${ }^{39}$

Preclinical evidence suggests that a multilevel neuroimmune response involving microglial astrocytes in the spinal cord ${ }^{6,48-50}$ and brain, ${ }^{51,52}$ as well as macrophages activation in the dorsal root ganglia, ${ }^{53,54}$ may be a major contributor to the pathogenesis and persistence of pain. Proinflammatory cytokines and brain-derived neurotrophic factor (BDNF), produced by activated glial cells and macrophages, stimulate nociceptive neurons, resulting in 
modulation of spinal cord synaptic transmission, central sensitization and enhancement of pain states. ${ }^{55-57}$ Furthermore, an intraspinal injection of activated microglia was associated with newly developed signs of neuropathic pain in rats. ${ }^{58}$ Astrocytes also play an important role in the initiation and maintenance of central sensitization. ${ }^{4,5}$ Hypertrophy and increased production of proinflammatory cytokines linked to allodynia and hyperalgesia, such as IL$1 \beta$ and chemokines (eg CXCL2), have been reported in astrocytes from animal models of neuropathic pain. ${ }^{59}$

Similarly, in vivo studies have provided evidence of neuronal, glial and endothelial cytokine receptors in brain and spinal cord cells. ${ }^{11,12,60}$ Moreover, neurotrophic factors, which are essential in the maintenance and regulation of neuronal regeneration after peripheral nervous system (PNS) and central nervous system (CNS) injury, also have an important impact on chronic pain associated with in vivo neuroinflammation states. NGF, BDNF, and glial cell line-derived neurotrophic factor (GNDF) are all significantly elevated during neuroinflammation. ${ }^{61}$ Increased expression of NGF receptors in macrophages, Schwann cells and mast cells results in the release of BDNF once nociceptors are activated, acting as a central regulator of pain. ${ }^{61}$ In neuropathic pain models, BDNF expression is high in DRG neurons, astrocytes, and glial cells. Therefore, BDNF concentration is increased in astrocytes, contributing to neuroinflammation-mediated neuropathic pain. ${ }^{61,62}$

\section{Chronic Pain Onset and Glial Cells Involvement: Clinical Evidence}

Clinical studies determining the involvement of glial cells in the initiation and maintenance of chronic pain are limited. Loggia et al identified increased levels of brain translocator protein (TSPO), a marker of glial activation, especially in the thalamus of patients with chronic lumbar pain by using integrated positron emission tomographynuclear magnetic resonance (PET-NMR) imaging and radioligand ${ }^{11} \mathrm{CPBR} 28 .{ }^{63}$ TSPO concentration was also elevated in the postcentral gyrus and paracentral lobe, which are the cortical representations of lumbar spine and leg. ${ }^{63}$ Nevertheless, previous studies have documented the presence of elevated levels of inflammatory mediators in spinal tissue and CSF of patients with degenerative disk disease, suggesting glial activation may also occur in the spinal cord. ${ }^{64,65}$ In a recent cross-sectional study, Albrecht et al reported higher levels of TSPO in the neuroforamina, which contains DRG and nerve root, in patients with pain from lumbar radiculopathy when compared to healthy volunteers. ${ }^{66}$ Based on the utility of functional magnetic resonance imaging (MRI) and ${ }^{18} \mathrm{~F}$-fluorodeoxyglucose 18F-FDG positron emission tomography (PET) on detecting active regions of the brain involved in pain, Zhou et al reviewed the ${ }^{18}$ F-FDG PET/CT scans of 13 patients with LBP and compared the normalized maximum standardized uptake value (SUVmax) with 13 control patients. SUVmax was used to determine the uptake of ${ }^{18} \mathrm{~F}$-FDG at different spine levels. Authors reported a significant increased SUVmax at T7, T8, T9, and T10 levels in patients with LBP in comparison with the control group. These findings suggest a regional increased metabolic activity in specific areas that could be targeted by SCS in patients with LBP. ${ }^{67}$

Recently, Palada et al reported a significant association between IL-8 levels in CSF, neuroinflammation and pain. In a prospective cohort study, the authors compared CSF, serum and disc-tissue cytokine levels from patients with DDD, LDH and healthy controls. A statistically significant correlation between serum and CSF concentrations of IL-8 and monocyte chemoattractant protein 1 (MCP1) was reported in patients with $\mathrm{LDH}$, and DDD respectively $(\mathrm{r}=0.489, \mathrm{p}=0.002$ and $\mathrm{r}=0.332, \mathrm{p}=0.037$ respectively). In addition, IL-6 serum levels were directly associated with leg pain intensity in patients with LDH whereas global pain intensity was correlated with CSF IL-8 levels in LDH male patients $(r=0.395 ; p=0.038)$. Similarly, serum IL-8 and MCP1 levels were correlated with back pain intensity in DDD female patients $(\mathrm{r}=$ $0.462, p=0.035$ and $(r=0.488 ; p=0.021$, respectively). Therefore, authors suggested that these cytokines play an essential role in neuroinflammation and pain as well as in the TSPO expression from intervertebral discs of patients with LDH and DDD. ${ }^{68}$

\section{Effects of Spinal Cord Stimulation and Modulation on Neuroinflammation}

\section{Preclinical Evidence}

Recent developments in bioelectronic medicine, SCS and neuromodulation devices have been associated with improved functional outcomes. A low-rate pulsed electric field applied into the spinal cord segments activates the A-beta fibers (mechanosensitive) in the neural network, deterring the nociceptive signaling carried out by A-delta and $\mathrm{C}$ fibers. Consequently, the brain will perceive paresthesia instead of pain. ${ }^{8,69,70}$ DRG stimulation (DRG-S), paresthesia-free SCS (PF-SCS), B-SCS, HF-SCS and the 
evoked compound action potential SCS (ECAP-SCS) are some of the novel SCS paradigms. Modulation of glial activation is one of the potential mechanisms by which SCS improves mechanical allodynia and neuropathic pain in the setting of chronic lumbar pain and persistent spinal pain syndrome. ${ }^{62,71}$ Sato et al reported a significant reduction in pain and glial activation measured by decreased microglial (ie immunostaining of OX-42) and astrocyte markers (ie glial fibrillary acidic protein or GFAP and MCP-1) with the use of superficial and deep dorsal horn lamina paresthesia-SCS (P-SCS) in animal models. ${ }^{72}$ Nerve injury was associated with bilateral OX-42 marker increase when compared to naïve controls. Both, $60 \mathrm{~Hz}$ and $4 \mathrm{~Hz}$ SCS frequencies, were shown to reduce OX-42 staining (ipsilateral $\mathrm{p}=0.0001$; contralateral $\mathrm{p}=0.001$ and ipsilateral $\mathrm{p}=0.0001$; contralateral $\mathrm{p}=0.003$, respectively) in comparison with the sham SCS control. Similar results were observed in astrocytes markers (GFAP and MCP-1). However, the greatest effect on pain relief was seen after 6 hour of $60 \mathrm{~Hz} \mathrm{SCS}{ }^{69}$ Likewise, an elevated expression of IL- $1 \beta$ and IL- 6 has been reported in rats after sparednerve injury, which reversed after 72 hours of continuous SCS, this effect being dose-dependent. ${ }^{73,74}$

Extensive research by Vallejo et al elucidated the molecular effects of continuous SCS in neural tissue and relevant changes in gene expression observed after SCS in neuropathic pain animal models. The use of P-SCS at $50 \mathrm{~Hz}$ during 72 hours allowed for modulation of genes involved in the onset of chronic neuropathic pain after neural injury, which had a significant impact on subsequent immune/inflammatory response by glial cells, neuron regeneration, and ions transport. ${ }^{75}$ However, the human glia/neuron ratio varies among spinal segments (eg 20:1 from segments T8-T11), resulting in different response to electrical stimulation. ${ }^{76}$ Therefore, SCS can be adapted based on targeted segments and the expected response. Vallejo's group recently provided important anatomical and functional evidence on the effects of customized SCS in neuropathic pain animal models. Authors reported that differential target multiplexed programming spinal cord stimulation (DTMP-SCS) better modulates neuroglia interaction, leading to greater thermal and mechanically induced pain relief when compared to low or high frequencies. ${ }^{77}$ In addition, this group identified more than 110 genes located in the spinal cord (and more than 100 in the DRG) by using conventional SCS. ${ }^{75}$ These genes were directly associated with previously described immune responses to neural injury and increased expression has been confirmed through isolation of their RNA sequences in different pain models such as spared-nerve injury and chronic constriction injury of the sciatic nerve. ${ }^{75,78}$

Activation of purinergic receptor P2X7 in SGC of DRG results in $\mathrm{P} 2 \mathrm{X} 3$ receptor downregulation and subsequent modulation of pathologic responses to painful stimuli in animal models. ${ }^{79}$ Therefore, the proximity of DRG to the DRG-S electrical field may allow for modulation of SGC activation. ${ }^{80}$ The transfer of the sensory impulses from the peripheral nerve receptor to the spinal cord occurs through the axonal synapses of cell bodies from primary somatosensory neurons in the DRG and neuronal somata. Structural and physiologic changes taking place within this pathway were described by Rozanski et al in a transglial transmission model, defined by the authors as "sandwich synapse". ${ }^{81}$

\section{Clinical Evidence}

To date, there is a paucity of clinical studies addressing the impact of current SCS modalities on pain intensity and/or neuroinflammation in patients with chronic LBP, radicular pain and FBSS. SCS has an important impact on the CSF concentration of several proteins involved in pain onset and intensity, synaptic plasticity, learning memory, nociceptive signaling, and immune regulation. ${ }^{82}$ In a matched control study, McCarthy et al compared the CSF concentration of BDNF, VEGF, and MCP-1 between patients diagnosed with chronic lumbar pain with or without FBSS. CFS concentrations of BDNF and MCP-1 were significantly higher in patients with FBSS when compared with their chronic lumbar pain matched controls $(\mathrm{p}=0.01$ and $\mathrm{p}=0.0001$ respectively). Moreover, authors reported a significant reduction in VEGF CSF concentrations after five minutes of SCS in the FBSS group $(p=0.01) .{ }^{83}$ Similarly, increased serum levels of IL-10, an antiinflammatory cytokine, have been found after B-SCS in patients who experienced pain level and clinical improvement whereas pro-inflammatory cytokine HMGB1 remained unchanged. ${ }^{84}$

Salivary concentration of inflammatory mediators has also been assessed in patients with CRPS. In a prospective single-center trial, Gravius et al correlated saliva and serum levels of HMGB-1, TNF- $\alpha$, IL-1 $\beta$, IL-6, IL-10 with clinical parameters (ie pain levels, mood, and sleep quality) in patients with CRPS eligible for L4-DRG-S. Authors reported a significant reduction in pain levels (mean numeric rating scale; pre-DRG $\mathrm{DRTIM}_{\mathrm{S}}: 74.90 \pm 16.3$ 
compared to one-week DRG $\mathrm{DTIM}_{\mathrm{ST}}: 42.50 \pm 13.18$ and threemonths DRG $_{\text {STIM }}: 46.65 \pm 27.52 ; \mathrm{p}=0.003$ ). At baseline and post L4 DRG-S, all pro-inflammatory markers were significantly higher in patients with CRPS when compared to controls. In contrast, levels of anti-inflammatory IL-10 were significantly reduced in patients with CRPS after L4 DRG-S when compared to healthy controls $(\mathrm{p}=0.0063){ }^{85}$ Potential immunomodulatory SCS effects offer new therapeutic alternatives to avoid the onset and progression of neuropathic pain in diseases with a neuroinflammatory component and phenotype. However, the sensitivity and specificity of serum and saliva inflammation markers have not been well established in patients with persistent spinal pain syndrome. Thus, heterogeneous data and its clinical application should be carefully interpreted.

\section{Spinal Cord Stimulation Modalities}

SCS has transformed the management of chronic back pain syndrome ${ }^{3,86-88}$ and its benefits are widely recognized among physicians who treat the heterogeneous syndromes of chronic LBP. ${ }^{86,87,89}$ Technical advancements in SCS are greatly improving patients' outcomes while changing our current understanding of its mechanism of action. $^{70,90}$ As new technological advancements emerge, previous theories describing the SCS mechanisms of action are being challenged. ${ }^{91}$ For instance, former proposed mechanisms describing the effect of SCS were based on limited local spinal responses only. However, current literature supports the evidence of supraspinal effects and alteration of glial cell derived neuroinflammatory mediators. ${ }^{14,70,90,92}$

Gate-control theory is the fundamental theory behind tonic and low frequency SCS. This theory proposed paresthesia or induction of $A \beta$ fiber activation within dorsal column neurons causing interference among chronic painful stimuli, thus allowing amelioration of chronic pain. ${ }^{9,91}$ Tonic SCS uses a frequency of $40-80 \mathrm{~Hz}$, a fixed pulse width of $200-450 \mu$ s, and adjustable current amplitudes based on patient comfort level. ${ }^{93}$

Recent studies have shown certain SCS modalities may exert their effect through a paresthesia-free mechanism with the possibility of curtailing neuroinflammatory and cytokine derived pathways. ${ }^{14,88}$ HF10 SCS functions with a smaller pulse width of $30 \mu$ s in the setting of a much higher frequency ${ }^{3}$ and has been associated with a significant reduction in reported pain and greater long-term efficacy when compared to tonic SCS. ${ }^{17,18,94}$ Stimulation of wide dynamic range (WDR) neurons, known to be overactive in conditions such as persistent spinal pain syndrome, is one of the main proposed HF10 SCS mechanisms of action. ${ }^{90}$ HF10 SCS, which is technically a higher energy form of tonic SCS, may be able to desensitize WDR neurons in chronic pain conditions bringing them closer to preinjury states. ${ }^{69,70,90}$ Nevertheless, the mechanism of desensitization is still up for debate with some evidence suggesting inflammatory cytokine and chemokine signaling interactions. ${ }^{14}$

Similarly, B-SCS is another novel stimulatory modality that has shown significant results in both, patient-reported pain measures and long-term pain suppression. ${ }^{88,94,95}$ Riddle et al observed that higher frequency stimuli delivered at $500 \mathrm{~Hz}, 40$ stimuli per second with an interspike interval of $1000 \mu \mathrm{s}$, decreased patient reported paresthesia by $75 \%$ in comparison with tonic SCS. ${ }^{20,95,96}$ In addition, B-SCS has shown greater efficacy in patients with refractory pain ${ }^{9,95}$ and modulation of the medial pathway, which involves some of the affective response to pain. ${ }^{70,90,97-99}$ However, the scope of these reports may be limited considering their small sample sizes, which emphasizes the need for multicenter randomized clinical trials. ${ }^{18,95,100}$

\section{Conclusion}

SCS programming may modify glial signaling through modulation of neuronal and glial cell activation, potentially improving clinical and functional outcomes in patients with chronic lumbar pain. However, the mechanisms through which SCS exerts its effects in patients with persistent spinal pain syndrome are yet to be determined.

Current and future clinical trials should elucidate whether specific inflammatory patterns are the most accurate way to define an individual SCS therapeutic mode based on the molecular biotype of a given patient.

\section{Acknowledgments}

The authors acknowledge Alicia Nahhas, B.S. from the Department of Anesthesiology at The Ohio State University, for her exceptional contribution during the final editing process of this article.

\section{Funding}

The authors declare that no grants/funding were involved in supporting this manuscript.

\section{Disclosure}

The Ohio State University have received grants for research activities from Medtronic Inc. and SPR therapeutics, Inc. in which MEV, JFD and TW have served as 
Investigators. In addition, TW have received funds from Medtronic, Inc. for consulting fees within the last year. The authors report no other conflicts of interest in this work.

\section{References}

1. Hart OR, Uden RM, McMullan JE, Ritchie MS, Williams TD, Smith BH. A study of National Health Service management of chronic osteoarthritis and low back pain. Prim Health Care Res Dev. 2015;16(2):157-166.

2. Dowell D, Haegerich TM, Chou R. CDC guideline for prescribing opioids for chronic pain-United States, 2016. JAMA 2016;315(15):1624-1645.

3. Dones I, Levi V. Spinal cord stimulation for neuropathic pain: current trends and future applications. Brain Sci. 2018;8(8):138.

4. Ji -R-R, Kawasaki Y, Zhuang Z-Y, Wen Y-R DI. Possible role of spinal astrocytes in maintaining chronic pain sensitization: review of current evidence with focus on $\mathrm{bFGF} / \mathrm{JNK}$ pathway. Neuron Glia Biol. 2006;2(4):259.

5. Ji R-R, Berta T, Nedergaard M. Glia and pain: is chronic pain a gliopathy? Pain ${ }^{\circledR}$. 2013;154:S10-S28.

6. Hains BC, Waxman SG. Activated microglia contribute to the maintenance of chronic pain after spinal cord injury. J Neurosci. 2006;26(16):4308-4317.

7. Grace PM, Hutchinson MR, Maier SF, Watkins LR. Pathological pain and the neuroimmune interface. Nat Rev Immunol. 2014;14 (4):217-231.

8. Melzack R, Wall PD. Pain mechanisms: a new theory. Science. 1965;150(3699):971-979.

9. Chakravarthy K, Richter H, Christo PJ, Williams K, Guan Y. Spinal cord stimulation for treating chronic pain: reviewing preclinical and clinical data on paresthesia-free high-frequency therapy. Neuromodulation. 2018;21(1):10-18.

10. Kapural L, Yu C, Doust MW, et al. Comparison of $10-\mathrm{kHz}$ high-frequency and traditional low-frequency spinal cord stimulation for the treatment of chronic back and leg pain: 24-month results from a multicenter, randomized, controlled pivotal trial. Neurosurgery. 2016;79(5):667-677.

11. Vitkovic L, Bockaert J, Jacque C. "Inflammatory" cytokines: neuromodulators in normal brain? $J$ Neurochem. 2000;74 (2):457-471.

12. Rothwell NJ, Hopkins SJ. Cytokines and the nervous system II: actions and mechanisms of action. Trends Neurosci. 1995;18 (3):130-136.

13. Bäckryd E, Lind A-L, Thulin M, Larsson A, Gerdle B, Gordh T. High levels of cerebrospinal fluid chemokines point to the presence of neuroinflammation in peripheral neuropathic pain: a cross-sectional study of 2 cohorts of patients compared with healthy controls. Pain. 2017;158(12):2487.

14. McCarthy K, McCrory C. Cerebrospinal fluid levels of glial cell-derived neurotrophic factor correlate with spinal cord stimulation frequency in patients with neuropathic pain: a preliminary report. Spinal Cord. 2014;52(2):S8-S10.

15. Vallejo RSW, Kelley C, et al. Neuron-glial inflammasome enhanced reversal by DTM-SCS relative to high rate and low rate SCS in a neuropathic pain model. American Society for Regional Anesthesiology and Pain Medicine (ASRA); November 14- 17,2019; New Orleans, LA Abstract \#480. 2019.

16. Vallejo RTD, Kelley C, et al. Proteomics of Differential Target Multiplexed-SCS applied to an animal model of neuropathic pain. American Society for Regional Anesthesiology and Pain Medicine (ASRA); November 14- 17,2019; New Orleans, LA Abstract \#509. 2019 .
17. Gill JS, Asgerally A, Simopoulos TT. High-frequency spinal cord stimulation at $10 \mathrm{kHz}$ for the treatment of complex regional pain syndrome: a case Series of patients with or without previous spinal cord stimulator implantation. Pain Pract. 2019;19 (3):289-294.

18. Kinfe TM, Pintea B, Link C, et al. High frequency $(10 \mathrm{kHz})$ or burst spinal cord stimulation in failed back surgery syndrome patients with predominant back pain: preliminary data from a prospective observational study. Neuromodulation. 2016;19 (3):268-275.

19. Al-Kaisy A, Palmisani S, Smith TE, et al. Long-term improvements in chronic axial low back pain patients without previous spinal surgery: a cohort analysis of $10-\mathrm{kHz}$ high-frequency spinal cord stimulation over 36 months. Pain Med. 2018;19(6):1219-1226.

20. De Ridder D, Plazier M, Kamerling N, Menovsky T, Vanneste S. Burst spinal cord stimulation for limb and back pain. World Neurosurg. 2013;80(5):642-649.

21. Harvey AM. Classification of chronic pain-descriptions of chronic pain syndromes and definitions of pain terms. Clin J Pain. 1995;11(2):163.

22. Slipman CW, Shin CH, Patel RK, et al. Etiologies of failed back surgery syndrome. Pain Med. 2002;3(3):200-214.

23. Thomson S. Failed back surgery syndrome-definition, epidemiology and demographics. Br J Pain. 2013;7(1):56-59.

24. Zhang, Y-G, Guo T-M, Guo X, Wu S-X. Clinical diagnosis for discogenic low back pain. Int J Biol Sci. 2009;5(7):647.

25. Schäfer A, Hall T, Briffa K. Classification of low back-related leg pain-a proposed patho-mechanism-based approach. Man Ther. 2009;14(2):222-230

26. Wang J, Markova D, Anderson DG, Zheng Z, Shapiro IM, Risbud MV. TNF- $\alpha$ and IL-1 $\beta$ promote a disintegrin-like and metalloprotease with thrombospondin type I motif-5-mediated aggrecan degradation through syndecan-4 in intervertebral disc. J Biol Chem. 2011;286(46):39738-39749.

27. Chan WC, Sze KL, Samartzis D, Leung VY, Chan D. Structure and biology of the intervertebral disk in health and disease. Orthopedic Clinics. 2011;42(4):447-464.

28. Boos N, Weissbach S, Rohrbach H, Weiler C, Spratt KF, Nerlich AG. Classification of age-related changes in lumbar intervertebral discs: 2002 Volvo Award in basic science. Spine. 2002;27(23):2631-2644.

29. Beaudreuil J, Dieude P, Poiraudeau S, Revel M. Disabling chronic low back pain with Modic type 1 MRI signal: acute reduction in pain with intradiscal corticotherapy. Ann Phys Rehabil Med. 2012;55(3):139-147.

30. Shamji MF, Setton LA, Jarvis W, et al. Proinflammatory cytokine expression profile in degenerated and herniated human intervertebral disc tissues. Arthritis Rheum. 2010;62(7):1974-1982.

31. Lee S, Moon CS, Sul D, et al. Comparison of growth factor and cytokine expression in patients with degenerated disc disease and herniated nucleus pulposus. Clin Biochem. 2009;42(15):1504-1511.

32. Burke J, Watson R, McCormack D, Dowling F, Walsh M, Fitzpatrick J. Intervertebral discs which cause low back pain secrete high levels of proinflammatory mediators. J Bone Joint Surg Br. 2002;84(2):196-201.

33. Goode AP, Carey TS, Jordan JM. Low back pain and lumbar spine osteoarthritis: how are they related? Curr Rheumatol Rep. 2013;15(2):305.

34. Igarashi A, Kikuchi S-I, Konno S-I. Correlation between inflammatory cytokines released from the lumbar facet joint tissue and symptoms in degenerative lumbar spinal disorders. J Orthopaedic Sci. 2007;12(2):154.

35. Amaya F, Samad TA, Barrett L, Broom DC, Woolf CJ. Periganglionic inflammation elicits a distally radiating pain hypersensitivity by promoting COX-2 induction in the dorsal root ganglion. Pain. 2009;142(1-2):59-67. 
36. Chahine M, O’Leary ME. Regulation/modulation of sensory neuron sodium channels. In: Voltage Gated Sodium Channels. Springer; 2014:111-135.

37. Li X-Q, Wang J, Fang B, Tan W-F MH. Intrathecal antagonism of microglial TLR 4 reduces inflammatory damage to blood-spinal cord barrier following ischemia/reperfusion injury in rats. $\mathrm{Mol}$ Brain. 2014;7(1):28.

38. Tremblay M-È, Stevens B, Sierra A, Wake H, Bessis A, Nimmerjahn A. The role of microglia in the healthy brain. J Neurosci. 2011;31(45):16064-16069.

39. Vezzani A, Viviani B. Neuromodulatory properties of inflammatory cytokines and their impact on neuronal excitability. Neuropharmacology. 2015;96:70-82.

40. Marin I, Kipnis J. Learning and memory ... and the immune system. Learning Memory. 2013;20(10):601-606.

41. Tian A, Ma H, Zhang R, et al. Interleukin17A promotes postoperative cognitive dysfunction by triggering $\beta$-amyloid accumulation via the transforming growth factor- $\beta$ (TGF $\beta) /$ smad signaling pathway. PLoS One. 2015;10(10):e0141596.

42. Feng $X$, Valdearcos $M$, Uchida $Y$, Lutrin D, Maze M, Koliwad SK. Microglia mediate postoperative hippocampal inflammation and cognitive decline in mice. JCI Insight. 2017;2:7.

43. Allan SM, Tyrrell PJ, Rothwell NJ. Interleukin-1 and neuronal injury. Nat Rev Immunol. 2005;5(8):629-640.

44. Montgomery SL, Bowers WJ. Tumor necrosis factor-alpha and the roles it plays in homeostatic and degenerative processes within the central nervous system. J Neuroimmune Pharmacol. 2012;7(1):42-59.

45. Xu J, Dong H, Qian Q, et al. Astrocyte-derived CCL2 participates in surgery-induced cognitive dysfunction and neuroinflammation via evoking microglia activation. Behav Brain Res. 2017;332:145-153.

46. Mika J, Zychowska M, Popiolek-Barczyk K, Rojewska E, Przewlocka B. Importance of glial activation in neuropathic pain. Eur J Pharmacol. 2013;716(1-3):106-119.

47. Chavan SS, Pavlov VA, Tracey KJ. Mechanisms and therapeutic relevance of neuro-immune communication. Immunity. 2017;46 (6):927-942.

48. Clark AK, Gentry C, Bradbury EJ, McMahon SB, Malcangio M. Role of spinal microglia in rat models of peripheral nerve injury and inflammation. Eur j Pain. 2007;11(2):223-230.

49. McMahon SB, La Russa F, Bennett DL. Crosstalk between the nociceptive and immune systems in host defence and disease. Nat Rev Neurosci. 2015;16(7):389-402.

50. Raghavendra V, Rutkowski MD, DeLeo JA. The role of spinal neuroimmune activation in morphine tolerance/hyperalgesia in neuropathic and sham-operated rats. J Neurosci. 2002;22(22):9980-9989.

51. Wei X-H, Wei X, Chen F-Y, et al. The upregulation of translocator protein $(18 \mathrm{kDa})$ promotes recovery from neuropathic pain in rats. J Neurosci. 2013;33(4):1540-1551.

52. Taylor AM, Mehrabani S, Liu S, Taylor AJ, Cahill CM. Topography of microglial activation in sensory-and affect-related brain regions in chronic pain. J Neurosci Res. 2017;95(6):1330-1335.

53. Lacor P, Benavides J, Ferzaz B. Enhanced expression of the peripheral benzodiazepine receptor $(\mathrm{PBR})$ and its endogenous ligand octadecaneuropeptide (ODN) in the regenerating adult rat sciatic nerve. Neurosci Lett. 1996;220(1):61-65.

54. Latremoliere A, Latini A, Andrews N, et al. Reduction of neuropathic and inflammatory pain through inhibition of the tetrahydrobiopterin pathway. Neuron. 2015;86(6):1393-1406.

55. Kawasaki Y, Zhang L, Cheng J-K, Ji -R-R. Cytokine mechanisms of central sensitization: distinct and overlapping role of interleukin-1 $\beta$, interleukin-6, and tumor necrosis factor- $\alpha$ in regulating synaptic and neuronal activity in the superficial spinal cord. J Neurosci. 2008;28(20):5189-5194.
56. Coull JA, Beggs S, Boudreau D, et al. BDNF from microglia causes the shift in neuronal anion gradient underlying neuropathic pain. Nature. 2005;438(7070):1017-1021.

57. Ji -R-R, Suter MR. p38 MAPK, microglial signaling, and neuropathic pain. Mol Pain. 2007;3:1744-8069-1743-1733.

58. Tsuda M, Shigemoto-Mogami Y, Koizumi S, et al. P2X 4 receptors induced in spinal microglia gate tactile allodynia after nerve injury. Nature. 2003;424(6950):778-783.

59. Chen G, Park C-K, Xie R-G, Berta T, Nedergaard M, Ji -R-R. Connexin-43 induces chemokine release from spinal cord astrocytes to maintain late-phase neuropathic pain in mice. Brain. 2014;137(8):2193-2209.

60. Vezzani A, French J, Bartfai T, Baram TZ. The role of inflammation in epilepsy. Nat Rev Neurol. 2011;7(1):31.

61. Pezet S, McMahon SB. Neurotrophins: mediators and modulators of pain. Annu Rev Neurosci. 2006;29:507-538.

62. Bjurstrom MF, Giron SE, Griffis CA. Cerebrospinal fluid cytokines and neurotrophic factors in human chronic pain populations: a comprehensive review. Pain Pract. 2016;16(2):183-203.

63. Loggia ML, Chonde DB, Akeju O, et al. Evidence for brain glial activation in chronic pain patients. Brain. 2015;138(3):604-615.

64. Wuertz K, Haglund L. Inflammatory mediators in intervertebral disk degeneration and discogenic pain. Global Spine J. 2013;3 (03):175-184.

65. Saal JS, Franson RC, Dobrow R, Saal JA, White AH, Goldthwaite N. High levels of inflammatory phospholipase A2 activity in lumbar disc herniations. Spine. 1990;15(7):674-678.

66. Albrecht D, Ahmed S, Kettner N, et al. Neuroinflammation of the spinal cord and nerve roots in chronic radicular pain patients. Pain. 2018;159(5):968.

67. Zhou X, Cipriano P, Kim B, et al. Detection of nociceptive-related metabolic activity in the spinal cord of low back pain patients using 18F-FDG PET/CT. Scandinavian J Pain. 2017;15:53-57.

68. Palada V, Ahmed AS, Finn A, Berg S, Svensson CI, Kosek E. Characterization of neuroinflammation and periphery-to-CNS inflammatory cross-talk in patients with disc herniation and degenerative disc disease. Brain Behav Immun. 2019;75:60-71.

69. Van Havenbergh T, Vancamp T, Van Looy P, Vanneste S, De Ridder D. Spinal cord stimulation for the treatment of chronic back pain patients: $500-\mathrm{Hz}$ vs. $1000-\mathrm{Hz}$ burst stimulation. Neuromodulation. 2015;18(1):9-12.

70. Barchini J, Tchachaghian S, Shamaa F, et al. Spinal segmental and supraspinal mechanisms underlying the pain-relieving effects of spinal cord stimulation: an experimental study in a rat model of neuropathy. Neuroscience. 2012;215:196-208.

71. Watkins LR, Milligan ED, Maier SF. Spinal cord glia: new players in pain. Pain. 2001;93(3):201-205.

72. Sato KL, Johanek LM, Sanada LS, Sluka KA. Spinal cord stimulation reduces mechanical hyperalgesia and glial cell activation in animals with neuropathic pain. Anesth Analg. 2014;118(2):464.

73. Tilley D, Vallejo R, Benyamin R, Vogel L, Kramer J. Acute spinal cord stimulation alters cytokine gene expression in the meninges. Fed Am Soc Exp Biol. 2009.

74. Tilley D, Benyamin R, Vallejo R Dose dependent changes in gene expression using spinal cord stimulation (SCS) In: North American Neuromodulation Society Meeting 2019. Las Vegas: North American Neuromodulation Society; 2019:2010.

75. Vallejo R, Tilley DM, Cedeño DL, Kelley CA, DeMaegd M, Benyamin R. Genomics of the effect of spinal cord stimulation on an animal model of neuropathic pain. Neuromodulation. 2016;19(6):576-586.

76. Ruiz-Sauri A, Orduña-Valls JM, Blasco-Serra A, et al. Glia to neuron ratio in the posterior aspect of the human spinal cord at thoracic segments relevant to spinal cord stimulation. J Anat. 2019;235(5):997-1006. 
77. Vallejo R, Kelley CA, Gupta A, Smith WJ, Vallejo A, Cedeño DL. Modulation of neuroglial interactions using differential target multiplexed spinal cord stimulation in an animal model of neuropathic pain. Mol Pain. 2020;16:1744806920918057.

78. Stephens KE, Chen Z, Sivanesan E, et al. RNA-seq of spinal cord from nerve-injured rats after spinal cord stimulation. Mol Pain. 2018;14:1744806918817429.

79. Chen Y, Zhang X, Wang C, Li G, Gu Y, Huang L-YM. Activation of $\mathrm{P} 2 \mathrm{X} 7$ receptors in glial satellite cells reduces pain through downregulation of $\mathrm{P} 2 \mathrm{X} 3$ receptors in nociceptive neurons. Proc Natl Acad Sci. 2008;105(43):16773-16778.

80. Pan B, Yu H, Fischer GJ, Kramer JM, Hogan QH. Dorsal root ganglionic field stimulation relieves spontaneous and induced neuropathic pain in rats. J Pain. 2016;17(12):1349-1358.

81. Rozanski GM, Li Q, Stanley EF. Transglial transmission at the dorsal root ganglion sandwich synapse: glial cell to postsynaptic neuron communication. Eur J Neurosci. 2013;37(8):1221-1228.

82. Lind AL, Emami Khoonsari P, Sjödin M, et al. Spinal cord stimulation alters protein levels in the cerebrospinal fluid of neuropathic pain patients: a proteomic mass spectrometric analysis. Neuromodulation. 2016;19(6):549-562.

83. McCarthy KF, Connor TJ, McCrory C. Cerebrospinal fluid levels of vascular endothelial growth factor correlate with reported pain and are reduced by spinal cord stimulation in patients with failed back surgery syndrome. Neuromodulation. 2013;16(6):519-522.

84. Kinfe TM, Muhammad S, Link C, Roeske S, Chaudhry SR, Yearwood TL. Burst spinal cord stimulation increases peripheral antineuroinflammatory interleukin 10 levels in failed back surgery syndrome patients with predominant back pain. Neuromodulation. 2017;20(4):322-330.

85. Gravius N, Chaudhry SR, Muhammad S, et al. Selective L4 dorsal root ganglion stimulation evokes pain relief and changes of inflammatory markers: part I profiling of saliva and serum molecular patterns. Neuromodulation. 2019;22(1):44-52.

86. Vallejo R, Bradley K, Kapural L. Spinal cord stimulation in chronic pain: mode of action. Spine. 2017;42:S53-S60.

87. Taylor RS, Desai MJ, Rigoard P, Taylor RJ. Predictors of pain relief following spinal cord stimulation in chronic back and leg pain and failed back surgery syndrome: a systematic review and meta-regression analysis. Pain Pract. 2014;14(6):489-505.

88. Sayed D, Kallewaard JW, Rotte A, Jameson J, Caraway D. Pain relief and improvement in quality of life with $10 \mathrm{kHz}$ SCS therapy: summary of clinical evidence. CNS Neurosci Ther. 2020;26(4):403-415.
89. Nissen M, Ikäheimo T-M, Huttunen J, Leinonen V. von Und Zu Fraunberg M. Long-term outcome of spinal cord stimulation in failed back surgery syndrome: 20 years of experience with 224 consecutive patients. Neurosurgery. 2019;84(5):1011-1018.

90. Kirketeig T, Schultheis C, Zuidema X, Hunter CW, Deer T. Burst spinal cord stimulation: a clinical review. Pain Med. 2019;20 (Supplement_1):S31-S40.

91. Mendell LM. Constructing and deconstructing the gate theory of pain. Pain ${ }^{\circledR}$. 2014;155(2):210-216.

92. Deer TR, Pope JE. Atlas of Implantable Therapies for Pain Management. Vol. 10. Springer; 2011.

93. Shechter R, Yang F, Xu Q, et al. Conventional and kilohertz-frequency spinal cord stimulation produces intensity-and frequency-dependent inhibition of mechanical hypersensitivity in a rat model of neuropathic pain Anesthesiology. 2013;119(2):422-432.

94. Ghosh PE, Simopolous TT. A review of the Senza System: a novel, high frequency $10 \mathrm{kHz}$ (HF10), paresthesia free spinal cord stimulator. Pain Manag. 2018;9(3):225-231.

95. De Ridder D, Perera S, Vanneste S. Are $10 \mathrm{kHz}$ stimulation and burst stimulation fundamentally the same? Neuromodulation. 2017;20(7):650-653.

96. De Ridder D, Vanneste S, Plazier M, van der Loo E, Menovsky T. Burst spinal cord stimulation: toward paresthesia-free pain suppression. Neurosurgery. 2010;66(5):986-990.

97. Rosenberg JC, Schultz DM, Duarte LE, Rosen SM, Raza A. Increased pain catastrophizing associated with lower pain relief during spinal cord stimulation: results from a large post-market study. Neuromodulation. 2015;18(4):277-284.

98. Osman A, Barrios FX, Gutierrez PM, Kopper BA, Merrifield T, Grittmann L. The Pain Catastrophizing Scale: further psychometric evaluation with adult samples. J Behav Med. 2000;23 (4):351-365.

99. McWilliams L, Asmundson G. Assessing individual differences in attention to pain: psychometric properties of the Pain Vigilance and Awareness Questionnaire modified for a non-clinical pain sample. Pers Individ Dif. 2001;31(2):239-246.

100. Muhammad S, Roeske S, Chaudhry SR, Kinfe TM. Burst or highfrequency $(10 \mathrm{kHz})$ spinal cord stimulation in failed back surgery syndrome patients with predominant back pain: one year comparative data. Neuromodulation. 2017;20(7):661-667.
Journal of Pain Research

\section{Publish your work in this journal}

The Journal of Pain Research is an international, peer reviewed, open access, online journal that welcomes laboratory and clinical findings in the fields of pain research and the prevention and management of pain. Original research, reviews, symposium reports, hypothesis formation and commentaries are all considered for publication. The manuscript management system is completely online and includes a very quick and fair peer-review system, which is all easy to use. Visit http:// www.dovepress.com/testimonials.php to read real quotes from published authors. 